\title{
TRANSEXUALISMO Y SALUD MENTAL
}

\author{
Felipe Hurtado ${ }^{1}$, MARCELino GÓMEZ ${ }^{2}$ y FranCisco DONAT ${ }^{1}$ \\ ${ }^{1}$ Centro de Salud Sexual y Reproductiva, Centro de salud Fuente San Luis, Valencia \\ ${ }^{2}$ Servicio de Endocrinología y Nutrición, Hospital Universitario Dr. Peset, Valencia
}

\begin{abstract}
Resumen: El objetivo principal de este estudio ha sido valorar la salud mental de las personas transexuales y, complementariamente, la frecuencia de agresiones de las que son objeto debido a la estigmatización del fenómeno en nuestra sociedad.La muestra estudiada consta de 26 sujetos, 13 hombres y 13 mujeres, seleccionados mediante muestreo consecutivo y que solicitaban iniciar el proceso de reasignación sexual. Todos cumplían los criterios diagnósticos establecidos en los manuales (DSM-IV-TR y CIE-10), también las normas de evaluación recomendadas por la Asociación Internacional «Harry Benjamin» respecto a los Trastornos de Identidad de Género.Los resultados han revelado una baja frecuencia de patología mental asociada y perfiles normales de personalidad, pero con niveles altos de estrés como consecuencia tanto del proceso de decisión y tiempo de espera para poder iniciar el tratamiento, como por la búsqueda de recursos al estar excluidos los tratamientos hormonales y quirúrgicos de las prestaciones sanitarias públicas.
\end{abstract}

Palabras clave: transexualismo, salud mental, evaluación psicológica, víctima de agresión.

\section{Transsexualism and mental health}

\begin{abstract}
The main objective of this study has been to evaluate the mental health of transsexual persons as well as the frequency of aggressions suffered by those due to the stigmatization of the phenomenon in our society. The study sample consisted of 26 subjects, 13 men and 13 women, selected by means of serial sampling among those that applied for initiation of sexual reassignment. All complied with the diagnostic criteria established by the manuals (DSM-IV-TR and CIE-10), as well as the evaluation norms recommended by the International Association «Harry Benjamin» regarding Gender Identity Disorders. The results reveal a low frequency of associate mental pathology and normal personality profiles, but high levels of stress as a consequence of both the decisionmaking process and the waiting time for treatment to begin, and the search of resources when finding one excluded from the hormonal and surgical treatments from public sanitary benefits.
\end{abstract}

Keywords: Transsexualism, mental health, psychological evaluation, aggression victim.

\section{INTRODUCCIÓN}

El transexualismo es la forma más extrema de la disforia de género, manifestando, la persona que lo padece, un sentimiento constante y una convicción persistente de pertenecer al sexo opuesto, hecho que le crea un permanente conflicto de identidad de género, siendo éste el aspecto cardinal del fenómeno (Tangpricha, Ducharme, Barber y Chipkin, 2003). Conse-

Recibido 13 junio 2006; aceptado 12 diciembre 2006.

Correspondencia: Felipe Hurtado Murillo, Centro de Salud Sexual y Reproductiva, Centro de Salud Fuente San Luis, C/ Arabista Ambrosio Huici, 30. 46013 Valencia. Correo-e: hurtado_fel@gva.es cuencia de lo anterior, la persona transexual siente un deseo intenso de vivir y de ser aceptada como un miembro del género opuesto al sexo biológico, por lo general solicita un deseo de modificar su cuerpo, mediante métodos hormonales y/o quirúrgicos, para hacerlo lo más congruente posible con el género sentido y deseado (Hengstschläger et al, 2003).

En la actualidad hay un debate abierto sobre si el transexualismo debería ser considerado un trastorno mental y si debería, por consiguiente, estar incluido en los manuales al uso de los trastornos mentales: manual diagnóstico y estadístico de los trastornos mentales, texto revisado (DSM-IV-TR) (APA, 2002) y la clasificación internacional de enfermedades (CIE, 10) 
(OMS, 1992). La Organización Mundial de la Salud (OMS, 1992), por su parte, considera que el transexualismo es un trastorno mental en la medida en que la manifestación produce un sufrimiento significativo y una ventaja desadaptativa importante.

El diagnóstico se realiza mediante criterios establecidos por la comunidad científica y que están establecidos tanto en el DSM-IV-TR (APA, 2002) como en la CIE-10 (OMS, 1992). Existen, no obstante, estudios críticos sobre la adecuación de estos manuales para establecer un correcto diagnóstico, como el realizado por Bower (2001), quien al analizar el DSM-IV encuentra que en la definición y descripción del trastorno de identidad de género, se omiten un número significativo de características importantes, estando los criterios aumentados y la sintomatología focalizada. Concluye que el manual es adecuado para el diagnóstico, pero que tiene aspectos que pueden impedir un diagnóstico exacto.

Desde hace varias décadas la experiencia contrastada de muchos investigadores de reconocido prestigio internacional, alerta sobre que los tratamientos intentados desde diferentes especialidades, se han mostrado ineficaces para reconciliar a la persona transexual con el sexo biológico de pertenencia. En concreto, la psicoterapia se ha mostrado ineficaz para intentar ajustar la mente al cuerpo.

En cambio el tratamiento de reasignación de género o ajustar el cuerpo a la mente, bien con hormonoterapia bien con cirugía o con ambas, si que ha demostrado que es el mejor camino para normalizar sus vidas, mientras que al mismo tiempo se reconoce la importancia de aplicar, previamente al tratamiento hormonal y/o quirúrgico irreversible, un tratamiento psicoterapéutico, no con la intención de curar el trastorno de identidad sexual, sino de ayudar a la persona a sentirse mejor dentro de su nueva identidad de género y a enfrentarse de forma eficaz a otros problemas no relacionados con el género (Vogt, 1968; Walinder y Thuwe, 1975; Walinder, Lundström y Thuwe, 1978; Rehman, Lazer, Benet, Schaefer y Melman, 1999; Michel, Mormont y Legros, 2001).

Asimismo, es de aceptación generalizada el apoyo psicoterapéutico después de la transfor- mación para ayudar a afrontar las dificultades emocionales, sociales y sexuales derivadas de su nueva apariencia (Calanca, 1991; Snaith, Tarsh y Reid, 1993; Rakic, Starcevic, Maric y Kelin, 1996; Cohen-Kettenis y van Goozen, 1997). Ahora bien, para realizar un óptimo tratamiento se precisa de la intervención de un equipo multidisciplinar que agrupe a diferentes especialistas y coordine las actuaciones si se quiere tener éxito en el tratamiento (Monstrey et al, 2001). El protocolo del proceso transexualizador que tiene más aceptación general entre los distintos equipos de tratamiento está basado en los estándares asistenciales propuestos desde la década de los años 80 por la Asociación Internacional Harry Benjamin de Trastornos de la Identidad de Género (HBIGDA, 1998, 2001), que recomienda la terapia triádica (psicológica, hormonal y quirúrgica), marcando criterios específicos de elegibilidad y adicionales de disposición de obligado cumplimiento tanto para la terapia hormonal como quirúrgica.

El presente estudio pretende clarificar diferentes aspectos relativos a la salud mental, reflejando las características más relevantes y la distribución por sexos, puesto que se hace necesario un análisis sobre si existe una psicopatología consustancial al propio trastorno de identidad de género $o$, si más bien, se trata de una reacción emocional a las circunstancias sociales adversas, a la falta de un abordaje sanitario multidisciplinar y la exclusión de la cartera de servicios del sistema nacional de salud. Complementariamente, analizamos la frecuencia de agresiones de las que suelen ser objeto debido, posiblemente, al rechazo social y a la estigmatización del fenómeno en nuestra sociedad.

\section{MÉTODO}

\section{Participantes}

La muestra estudiada está compuesta por 26 personas, 13 hombres y 13 mujeres, con edades comprendidas para los hombres entre los $18 \mathrm{y}$ 49 años (media $=28,92 ; D T=10,49)$ y para las mujeres entre los 17 y 40 años (media $=25,00$; $D T=7,33)$, que solicitaron atención psicológi- 
ca en el Centro de Salud Sexual y Reproductiva del Centro de Salud Fuente San Luis de Valencia dependiente de la Agencia Valenciana de Salud, por manifestar una identidad sexual/identidad de género discordante con su sexo biológico y solicitaban iniciar el proceso de cambio de sexo como forma más adecuada de resolver su conflicto de identidad y mejorar su calidad de vida.

La selección de los sujetos se ha realizado mediante muestreo consecutivo desde el año 2000. Siendo el criterio de selección el cumplir tanto los criterios diagnósticos establecidos en los manuales al uso [DSM-IV-TR (APA, 2002) y CIE-10 (OMS, 1992)] como los estándares asistenciales recomendados por la Asociación Internacional «Harry Benjamin» sobre trastornos de identidad de género (HBIGDA, 2001).

\section{Instrumentos}

Es necesario hacer un buen diagnóstico con un correcto diagnóstico diferencial, ya que la falta de certeza es un factor predictivo de arrepentimiento tras la realización del tratamiento de reasignación sexual y de la evolución posterior. El diagnóstico diferencial debe hacerse con el transvestismo fetichista, el transvestismo no fetichista, la orientación sexual egodistónica, el trastorno de la maduración sexual y con el trastorno sexual y de la identidad sexual no especificado. Para realizar un diagnóstico certero y hacer un correcto diagnóstico diferencial, nos hemos basado en criterios establecidos por la comunidad científica, tanto en el manual diagnóstico y estadístico de los trastornos mentales DSM-IV-TR (APA, 2002) como en la clasificación internacional de enfermedades CIE-10 (OMS, 1992).

Para cumplir los criterios específicos de elegibilidad y los adicionales de disposición, establecidos en los estándares propuestos por la Asociación Internacional Harry Benjamin sobre trastornos de identidad de género (HBIGDA, 1998), de necesario cumplimiento para iniciar tanto la terapia hormonal como quirúrgica, hemos utilizado entrevistas semiestructuradas para obtener los datos necesarios sobre antece- dentes de salud, datos biográficos, aparición y evolución del sentimiento de identidad sexual/identidad de género, orientación y conducta sexual, relación de pareja y datos familiares (Cano y Bergero, 2003). Además hemos utilizado las siguientes pruebas:

Escala Autoaplicada para la Evaluación del Estrés-Apoyo Social (Departamento de Salud Mental de California, 1981; adaptación española de Conde y Franch, 1984). La escala consta de dos partes. En la primera se explora el nivel de estrés en los últimos doce meses en las áreas familiar (6 ítems), económico-laboral (4 ítems) y personal (9 ítems). Los puntos de corte propuestos por niveles de estrés son: bajo (menos de 10 puntos), moderado (de 10 a 15 ) y alto (16 ó más).

En la segunda parte se explora el nivel de apoyo social del sujeto, relacionándolo con el nivel de frecuencia y calidad de sus relaciones afectivas y sociales. Consta de cinco ítems con cuatro alternativas de respuesta cada uno. Los puntos de corte por niveles son: bajo (menos de 15 puntos), moderado (de 15 a 29 ) y alto (30 ó más).

Inventario para la Depresión de Beck BDI (Beck, Rush, Shaw y Emery, 1979). Se trata de un instrumento de autoinforme que consta de 21 ítems (rango: 0-63 puntos) y mide la intensidad de los síntomas depresivos. El punto de corte más utilizado para discriminar entre la población sana de aquella que manifiesta síntomas depresivos es de 18. En sus ítems se da más importancia a los componentes cognitivos de la depresión que a los conductuales y somáticos. El coeficiente de fiabilidad por el método de las dos mitades es de 0,93 y desde la perspectiva de la validez convergente, la correlación con la evaluación clínica de la depresión oscila de 0,62 a 0,66.

Escala de Autoestima (Rosenberg, 1965; versión española de Echeburúa y Corral, 1998). Es un instrumento de autoinforme que consta de 10 ítems (rango: 10-40 puntos) y que mide el grado de satisfacción que una persona tiene consigo misma. La mitad de los ítems están planteados de forma afirmativa y la otra mitad de forma negativa, con el objetivo de controlar la aquiescencia. Cuanto mayor es la puntuación, 
mayor es el grado de autoestima. El punto de corte en la población adulta, según el estudio de Ward (1977), es de 29.

Cuestionario de Ansiedad Estado-Rasgo STAI-E/R (Spielberger, Gorsuch y Lushene, 1970). (Versión española de TEA, 1982). El inventario de ansiedad estado-rasgo es una prueba de autoinforme que consta de 20 ítems relacionados con la ansiedad-rasgo y de otros 20 relacionados con la ansiedad estado. El rango de las puntuaciones es de 0 a 60 en cada escala. La fiabilidad test-retest es de 0,81 en la escala de ansiedad-rasgo mientras que en la escala de ansiedad-estado es de 0,40. La consistencia interna oscila de 0,83 a 0,92 y la validez convergente con otras medidas de ansiedad oscila entre 0,58 y 0,79 . Para la caracterización de los sujetos hemos utilizado los baremos correspondientes a la población española donde al igual que en otras escalas medidas en percentiles, el punto de corte para el nivel alto se sitúa en 75 , el nivel bajo en $25 \mathrm{y}$ el moderado entre ambos.

Inventario de Situaciones y Respuestas de Ansiedad (ISRA; Miguel y Cano, 1986). Es un instrumento que evalúa la ansiedad en su triple sistema de respuestas: cognitiva, fisiológica y motora, ante situaciones de la vida cotidiana., es por ello que esta formado por tres subescalas con 22 situaciones concretas y una situación abierta que se utiliza solamente cuando existe una situación especialmente perturbadora para el sujeto y no está incluida en las otras 22 . La frecuencia de manifestación de las distintas respuestas de ansiedad se evalúan según una escala tipo Likert de cinco puntos $(0=$ casi nunca, $1=$ pocas veces, $2=$ unas veces si, unas veces no, 3 = muchas veces, $4=$ casi siempre). Las puntuaciones directas se convierten en centiles utilizando las tablas baremadas correspondientes, existiendo la posibilidad de utilizar baremos de población general y baremos de muestras clínicas. Los sujetos según percentil obtenido se clasifican en varios niveles de ansiedad (mínima, moderada, severa y extrema). Para la caracterización de los sujetos hemos utilizado los baremos correspondientes a la población general.

Cuestionario de Personalidad de Eysenck EPI (Eysenck y Eysenck, 1964). (Versión española de TEA, 1987). Este cuestionario es una prueba de autoinforme con respuesta SI-NO, que explora dos factores ortogonales y bipolares claramente definidos en la mayoría de los estudios: neuroticismo y extroversión. Además, tiene una escala de sinceridad con la que se puede identificar a los sujetos que tienden a responder en una dirección «deseable». Tiene dos formas paralelas (A y B) que permiten el retest en un tratamiento experimental, sin la interferencia de los factores de memoria. En esta investigación hemos utilizado la forma B. El punto de corte para la población adulta femenina (baremo profesional) en Neuroticismo es 13, en Extroversión 15 y en Sinceridad está entre 7 y 8 (todos ellos correspondientes al percentil 75). El punto de corte para la población adulta masculina (baremo profesional) en Neuroticismo es 9, en Extroversión es 16 y en Sinceridad está entre 7 y 8 (correspondientes al percentil 75).

Inventario Multifásico de Personalidad de Minnesota-2 (MMPI-2; Hathaway y McKinley, 1942. Adaptación española de Ávila-Espada y Jiménez-Gómez. TEA ediciones 2000). Partiendo del original MMPI publicado en 1942, Butcher hizo una revisión profunda a la nueva versión MMPI-2 en 1989 y en España realizaron la adaptación Alejando Ávila Espada y Fernando Jiménez Gómez de la Universidad de Salamanca. La prueba consta de 567 ítems con dos posibilidades de respuesta (verdadero-falso) y evalúa varios factores o aspectos de la personalidad a través de tres grupos de escalas: básicas, de contenido y suplementarias. Contiene también un grupo de escalas referentes a la validez y otras adicionales. Las interpretaciones de las escalas clínicas tienden a dirigirse a las elevaciones las puntuaciones en las distintas escalas. Para la interpretación, las puntuaciones directas se transforman en puntuaciones T que se obtienen en las tablas baremadas, siendo el punto de corte entre la elevaciones normales y las anormales las puntuaciones T 65 o superiores.

Escala de Miedo a la Evaluación Negativa (FNE; Watson y Friend, 1969). Esta escala tiene por objetivo evaluar el componente cognitivo de la ansiedad social. Consta de 30 ítems (rango: 0-30), con dos posibilidades de respuesta (verdadero o falso). El punto de corte propuesto para discriminar entre la población sana 
de la aquejada de ansiedad social es de 22-24 (Heimberg, Dodge, Hope, Kennedy, Zollo y Becker, 1990; Mattick, Peters y clarke, 1989). Escala de Estrés y Evitación Social (SAD; Watson y Friend, 1969). Se trata de una escala que consta de 28 ítems (rango: 0-28 puntos), con dos posibilidades de respuesta (verdadero o falso). 14 ítems se refieren al malestar subjetivo experimentado en situaciones sociales y otros 14 ítems a la evitación activa o deseo de evitación de dichas situaciones. El punto de corte propuesto para discriminar entre la población sana de la aquejada de ansiedad social es de 18-20 (Butler, Cullington, Munby, Amies y Gelder, 1984; Heimberg, Dodge, Hope, Kennedy, Zollo y Becker, 1990).

\section{Procedimiento}

La recogida de datos fue realizada durante las sesiones de evaluación diagnóstica y psicoterapéutica, bien de forma individual o bien con algún miembro de la familia, generalmente la madre o el padre, y en aquellos/as que tenían pareja estable, en ocasiones con la presencia de la pareja. La sesiones clínicas se pautaron con intervalos semanales o quincenales, requiriendo un mínimo de tres meses de contacto psicoterapéutico para completar el diagnóstico y poder iniciar la terapia hormonal. A partir de entonces mantienen un contacto regular para ayudar a afrontar las dificultades emocionales, sociales y sexuales derivadas de su nueva apariencia.

\section{Análisis de datos}

El análisis de datos se ha realizado con el programa estadístico SPSS 12.0 y ha consistido en la descripción de variables cualitativas y cuantitativas, cuantificación de frecuencias y transformación de los datos brutos en porcentajes. Para la comparación de resultados entre sexos en relación a las variables relativas a la salud mental, factores de personalidad y agresiones sexuales, hemos utilizado la prueba estadística de Chi-cuadrado de Pearson.

\section{RESULTADOS}

Las características relativas a las variables biográficas: sexo, estado civil, nivel cultural y profesión, aparecen reflejadas en la Tabla 1. Los datos globales de la muestra reflejan un reparto igualitario entre sexos biológicos con una edad media de 27 años, donde predomina la soltería, con un nivel cultural medio y en la mitad de los casos trabajando como asalariados/as. Debido a la estigmatización y rechazo social que en nuestra sociedad produce el transexualismo, a continuación se muestran las diversas agresiones sociales sufridas por las personas transexuales de la muestra estudiada (Tabla 2).

La mitad de la muestra sin distinción se sexo ha sufrido agresiones verbales $(53,8 \%$ de los hombres y $46,2 \%$ de las mujeres). La tasa de agresiones físicas para los hombres ha sido importante $(46,2 \%)$, en cambio, ha sido escasa $(7,7 \%)$ para las mujeres. Respecto a las agresiones sexuales, dos hombres biológicos las han sufrido frente a ninguna mujer. Por lo tanto, ni las agresiones verbales ni las sexuales muestran diferencias significativas entres sexos, en cambio, las agresiones físicas sufridas por los hombres biológicos reflejan una diferencia estadísticamente significativa respecto a las mujeres biológicas $(\mathrm{p}<0,05)$.

Pasando a describir las variables relativas a la salud mental (Tablas 3 y 4), los resultados según las variables analizadas expresan, que para el nivel de estrés en los últimos 12 meses, antes de iniciar el protocolo de evaluación psicodiagnóstica, la mitad de la muestra sin distinción de sexo tuvo un nivel de estrés alto, superando ligeramente, pero sin diferencias significativas, en tasa los hombres $(53,8 \%)$ a las mujeres $(46,1 \%)$, en cambio se igualaron a nivel moderado $(15,4 \%)$.

El apoyo social muestra que la mayoría lo siente tener a nivel moderado $(61,5 \%)$, mejorando la tasa en los hombres $(76,9 \%)$ frente a las mujeres $(46,1 \%)$, aunque las diferencias mostradas no alcanzan diferencias estadísticamente significativas.

La variable estado de ánimo expresa en cifras que la mitad de la muestra en su conjunto tiene un estado de ánimo normal, habiendo 
Tabla 1. Características biográficas según sexo biológico

\begin{tabular}{lcccccc}
\hline \multicolumn{1}{c}{ Variables } & \multicolumn{2}{c}{ Hombres } & \multicolumn{2}{c}{ Mujeres } & \multicolumn{2}{c}{ Total } \\
& $N$ & $(\%)$ & $N$ & $(\%)$ & $N$ & $(\%)$ \\
\hline Sexo biológico & 13 & $(50,0)$ & 13 & $(50,0)$ & 26 & $(100,0)$ \\
& & & & & & \\
Estado civil & & & & & & \\
$\quad$ Soltero/a & $(69,2)$ & 9 & $(69,2)$ & 18 & $(69,2)$ \\
$\quad$ Casado/a-conviviente & 2 & $(15,4)$ & 4 & $(30,8)$ & 6 & $(23,1)$ \\
Separado/a-divorciado/a & 2 & $(15,4)$ & 0 & $(0,0)$ & 2 & $(7,7)$ \\
Nivel cultural & & & & & & \\
Primarios & 4 & $(30,8)$ & 4 & $(30,8)$ & 8 & $(30,8)$ \\
Medios & 3 & $(23,1)$ & 8 & $(61,5)$ & 11 & $(42,3)$ \\
Superiores & 6 & $(46,1)$ & 1 & $(7,7)$ & 7 & $(26,9)$ \\
& & & & & & \\
Profesiones & & & & & \\
Patrón & & $(0,0)$ & 1 & $(7,7)$ & 1 & $(3,8)$ \\
Profesional & & $(15,4)$ & 1 & $(7,7)$ & 3 & $(11,5)$ \\
Asalariado/a & 2 & $(46,1)$ & 7 & $(53,8)$ & 13 & $(50,0)$ \\
Parado/a & 6 & $(23,1)$ & 2 & $(15,4)$ & 5 & $(19,2)$ \\
$\quad$ Estudiante & 3 & $(15,4)$ & 2 & $(15,4)$ & 4 & $(15,4)$ \\
\hline
\end{tabular}

Tabla 2. Agresiones sociales según sexo biológico

\begin{tabular}{|c|c|c|c|c|c|c|c|}
\hline \multirow[t]{2}{*}{ Variables } & \multicolumn{2}{|c|}{ Hombres } & \multicolumn{2}{|c|}{ Mujeres } & \multicolumn{2}{|c|}{ Total } & \multirow[b]{2}{*}{$\chi^{2}$} \\
\hline & $N$ & $(\%)$ & $N$ & $(\%)$ & $N$ & $(\%)$ & \\
\hline Agresiones verbales & & & & & & & 0,15 \\
\hline $\mathrm{Si}$ & 7 & $(53,8)$ & 6 & $(46,2)$ & 13 & $(50,0)$ & \\
\hline No & 6 & $(46,2)$ & 7 & $(53,8)$ & 13 & $(50,0)$ & \\
\hline Agresiones fisicas & & & & & & & $4,88^{*}$ \\
\hline $\mathrm{Si}$ & 6 & $(46,2)$ & 1 & $(7,7)$ & 7 & $(26,9)$ & \\
\hline No & 7 & $(53,8)$ & 12 & $(92,3)$ & 19 & $(73,1)$ & \\
\hline Agresiones sexuales & & & & & & & $2,16^{*}$ \\
\hline $\mathrm{Si}$ & 2 & $(15,4)$ & 0 & $(0,0)$ & 2 & $(7,7)$ & \\
\hline No & 11 & $(84,6)$ & 13 & $(100,0)$ & 24 & $(92,3)$ & \\
\hline
\end{tabular}

$* p<0,05$

un escaso porcentaje $(23,1 \%)$ tanto de hombres como de mujeres con depresión leve y otro tanto con depresión moderada $(23,1 \%$ para los hombres y $30,8 \%$ para las mujeres). No habiendo ninguna persona con nivel de depresión grave. Esta variable vuelve a mostrar que no existen diferencias significativas entre los sexos, a pesar de las diferencias encontradas.

La autoestima, medida en niveles, muestra que la mayoría de hombres $(76,9 \%)$ la tiene alta, en cambio para las mujeres los datos indican que hay ligeramente más mujeres $(53,8 \%)$ con baja autoestima que con alta $(46,2 \%)$, pero sin llegar a alcanzar nivel de significación estadística.

La medida de los niveles de ansiedad no refleja diferencias significativas, manifiesta que hay un alrededor de un tercio de la muestra $(38,5 \%)$ con nivel alto y una tasa igual con nivel moderado $(38,5 \%)$, habiendo solamente una mujer con ansiedad extrema.

Las medidas realizadas sobre la existencia de ansiedad social y evitación de situaciones socia- 
Tabla 3. Salud mental (I) según sexo biológico

\begin{tabular}{|c|c|c|c|c|c|c|c|}
\hline \multirow[t]{2}{*}{ Variables } & \multicolumn{2}{|c|}{ Hombres } & \multicolumn{2}{|c|}{ Mujeres } & \multicolumn{2}{|c|}{ Total } & \multirow[b]{2}{*}{$\chi^{2}$} \\
\hline & $N$ & $(\%)$ & $N$ & $(\%)$ & $N$ & $(\%)$ & \\
\hline $\begin{array}{l}\text { Nivel de estrés en } \\
\text { los últimos } 12 \text { meses }\end{array}$ & & & & & & & 0,18 \\
\hline Bajo & 4 & $(30,8)$ & 5 & $(38,5)$ & 9 & $(34,6)$ & \\
\hline Moderado & 2 & $(15,4)$ & 2 & $(15,4)$ & 4 & $(15,4)$ & \\
\hline Alto & 7 & $(53,8)$ & 6 & $(46,1)$ & 13 & $(50,0)$ & \\
\hline Apoyo social & & & & & & & 3,5 \\
\hline Bajo & 3 & $(23,1)$ & 5 & $(38,5)$ & 8 & $(30,8)$ & \\
\hline Moderado & 10 & $(76,9)$ & 6 & $(46,1)$ & 16 & $(61,5)$ & \\
\hline Alto & 0 & $(0,0)$ & 2 & $(15,4)$ & 2 & $(7,7)$ & \\
\hline Estado de ánimo & & & & & & & 0,22 \\
\hline Depresión ausente & 7 & $(53,8)$ & 6 & $(46,1)$ & 13 & $(50,0)$ & \\
\hline Depresión leve & 3 & $(23,1)$ & 3 & $(23,1)$ & 6 & $(23,1)$ & \\
\hline Depresión moderada & 3 & $(23,1)$ & 4 & $(30,8)$ & 7 & $(26,9)$ & \\
\hline Depresión grave & 0 & $(0,0)$ & 0 & $(0,0)$ & 0 & $(0,0)$ & \\
\hline Autoestima & & & & & & & 2,6 \\
\hline Baja & 3 & $(23,1)$ & 7 & $(53,8)$ & 10 & $(38,5)$ & \\
\hline Alta & 10 & $(76,9)$ & 6 & $(46,2)$ & 16 & $(61,5)$ & \\
\hline
\end{tabular}

les, muestran que solamente hay dos personas, una de cada sexo con este tipo de problema. La mayoría $(92,3 \%)$ obtiene puntuaciones de población sana.

Finalmente, sobre la existencia de miedo a situaciones sociales donde se pudiera descubrir el sexo biológico y por tanto su evitación siempre o la mayor parte de las veces, denota que todas la mujeres lo manifiestan y casi la mitad de los hombres $(46,1 \%)$, siendo esta diferencia entre sexos estadísticamente significativa $(p<0,01)$.

Del mismo modo que se han medido distintas variables de la salud mental, también se han medido distintos factores de la personalidad (Tablas 5 y 6). La ansiedad como rasgo de personalidad muestra que el $50 \%$ de la muestra en su conjunto lo manifiesta a niveles altos, con cercanas proporciones entre ambos sexos. En consonancia, la tasa global con altos niveles de neuroticismo o inestabilidad emocional es similar (46,2\%), aunque la distribución porcentual entre sexos expresa que hay dos tercios menos de mujeres que de hombres $(23,1 \%$ frente a $69,2 \%$ respectivamente), pero sin llegar a alcanzar diferencias significativas. La variable extroversión muestra que el $73 \%$ de la muestra es bien moderada bien alta en esta variable y el $27 \%$ es introvertida, no habiendo diferencias importantes entre los sexos.

La interpretación de resultados del estudio multifásico de la personalidad muestra en sus escalas clínicas, que la mayoría de las personas transexuales presentan perfiles normales y sin diferencias significativas entre los sexos, salvo en la escala masculinidad-feminidad donde alcanzan puntuaciones clínicamente significativas en el $69,2 \%$ de la muestra global, con mayor tasa en los hombres biológicos $(84,6 \%)$ que en las mujeres $(53,8 \%)$, pero sin que las diferencias mostradas entre sexos alcancen nivel de significatividad estadística. Datos que expresan conflictos sobre la identidad sexual y rechazo a los papeles tradicionales del propio sexo biológico.

Analizando pormenorizadamente las categorías clínicas, los datos sobre Hipocondría o preocupación excesiva por la salud muestran como solamente un hombre y dos mujeres alcanzan puntuaciones clínicamente significativas. La escala sobre Depresión que refleja no sólo los sentimientos de desánimo, pesimismo y desesperación que caracterizan el estado clínico de las personas depresivas, sino también caracte- 
Tabla 4. Salud mental (II) según sexo biológico

\begin{tabular}{|c|c|c|c|c|c|c|c|}
\hline \multirow[t]{2}{*}{ Variables } & \multicolumn{2}{|c|}{ Hombres } & \multicolumn{2}{|c|}{ Mujeres } & \multicolumn{2}{|c|}{ Total } & \multirow[b]{2}{*}{$\chi^{2}$} \\
\hline & $N$ & $(\%)$ & $N$ & $(\%)$ & $N$ & $(\%)$ & \\
\hline Ansiedad estado & & & & & & & 0,8 \\
\hline Baja & 3 & $(23,1)$ & 3 & $(23,1)$ & 6 & $(23,1)$ & \\
\hline Moderada & 4 & $(30,8)$ & 6 & $(46,1)$ & 10 & $(38,5)$ & \\
\hline Alta & 6 & $(46,1)$ & 4 & $(30,8)$ & 10 & $(38,5)$ & \\
\hline Ansiedad cognitiva & & & & & & & 2,25 \\
\hline Baja & 4 & $(30,8)$ & 5 & $(38,5)$ & 9 & $(34,6)$ & \\
\hline Moderada & 3 & $(23,1)$ & 4 & $(30,8)$ & 7 & $(26,9)$ & \\
\hline Alta & 6 & $(46,1)$ & 3 & $(23,1)$ & 9 & $(34,6)$ & \\
\hline Extrema & 0 & $(0,0)$ & 1 & $(7,7)$ & 1 & $(3,8)$ & \\
\hline Ansiedad fisiológica & & & & & & & 0,9 \\
\hline Baja & 4 & $(30,8)$ & 4 & $(30,8)$ & 8 & $(30,8)$ & \\
\hline Moderada & 4 & $(30,8)$ & 6 & $(46,1)$ & 10 & $(38,5)$ & \\
\hline Alta & 5 & $(38,5)$ & 3 & $(23,1)$ & 8 & $(30,8)$ & \\
\hline Ansiedad motora & & & & & & & 1,61 \\
\hline Baja & 4 & $(30,8)$ & 5 & $(38,5)$ & 9 & $(34,6)$ & \\
\hline Moderada & 4 & $(30,8)$ & 4 & $(30,8)$ & 8 & $(30,8)$ & \\
\hline Alta & 5 & $(38,5)$ & 3 & $(23,1)$ & 8 & $(30,8)$ & \\
\hline Extrema & 0 & $(0,0)$ & 1 & $(7,7)$ & 1 & $(3,8)$ & \\
\hline Ansiedad global & & & & & & & 2,51 \\
\hline Baja & 3 & $(23,1)$ & 4 & $(30,8)$ & 7 & $(53,8)$ & \\
\hline Moderada & 5 & $(38,5)$ & 6 & $(46,1)$ & 11 & $(42,3)$ & \\
\hline Alta & 5 & $(38,5)$ & 2 & $(15,4)$ & 7 & $(26,9)$ & \\
\hline Extrema & 0 & $(0,0)$ & 1 & $(7,7)$ & 1 & $(3,8)$ & \\
\hline Ansiedad social cognitiva & & & & & & & 0,00 \\
\hline Población sana & 12 & $(92,3)$ & 12 & $(92,3)$ & 24 & $(92,3)$ & \\
\hline Ansiedad social & 1 & $(7,7)$ & 1 & $(7,7)$ & 2 & $(7,7)$ & \\
\hline Estrés y evitación social & & & & & & & 0,00 \\
\hline Población sana & 12 & $(92,3)$ & 12 & $(92,3)$ & 24 & $(92,3)$ & \\
\hline Ansiedad social & 1 & $(7,7)$ & 1 & $(7,7)$ & 2 & $(7,7)$ & \\
\hline $\begin{array}{l}\text { Miedo a situaciones sociales } \\
\text { donde se pudiera descubrir } \\
\text { su sexo biológico }\end{array}$ & & & & & & & $9,57 * *$ \\
\hline Sin miedo & 7 & $(53,8)$ & 0 & $(0,0)$ & 7 & $(26,9)$ & \\
\hline Con miedo & 6 & $(46,1)$ & 13 & $(100,0)$ & 19 & $(73,1)$ & \\
\hline
\end{tabular}

$* * p<0,01$

rísticas básicas de la personalidad, tales como hiperresponsabilidad, valores personales rígidos y auto-castigo muestra datos idénticos a la escala anterior, es decir, un hombre y dos mujeres con perfil clínicamente significativo.
La tercera escala de Histeria de conversión muestra que en el total de la muestra solamente hay 4 personas $(15,4 \%$ de la muestra), dos hombres y dos mujeres, que alcanzan puntuaciones clínicamente significativas. 
Tabla 5. Factores de personalidad (I) según sexo biológico

\begin{tabular}{|c|c|c|c|c|c|c|c|}
\hline \multirow[t]{2}{*}{ Variables } & \multicolumn{2}{|c|}{ Hombres } & \multicolumn{2}{|c|}{ Mujeres } & \multicolumn{2}{|c|}{ Total } & \multirow[b]{2}{*}{$\chi^{2}$} \\
\hline & $N$ & $(\%)$ & $N$ & $(\%)$ & $N$ & $(\%)$ & \\
\hline Ansiedad rasgo & & & & & & & 1,18 \\
\hline Baja & 4 & $(30,8)$ & 5 & $(38,5)$ & 9 & $(34,6)$ & \\
\hline Moderada & 3 & $(23,1)$ & 1 & $(7,7)$ & 4 & $(15,4)$ & \\
\hline Alta & 6 & $(46,1)$ & 7 & $(53,8)$ & 13 & $(50,0)$ & \\
\hline Neuroticismo & & & & & & & 6,0 \\
\hline Bajo & 1 & $(7,7)$ & 1 & $(7,7)$ & 2 & $(7,7)$ & \\
\hline Moderado & 3 & $(23,1)$ & 9 & $(69,2)$ & 12 & $(46,2)$ & \\
\hline Alto & 9 & $(69,2)$ & 3 & $(23,1)$ & 12 & $(46,2)$ & \\
\hline Extroversión & & & & & & & 3,0 \\
\hline Baja & 3 & $(23,1)$ & 4 & $(30,8)$ & 7 & $(26,9)$ & \\
\hline Moderada & 9 & $(69,2)$ & 5 & $(38,5)$ & 14 & $(53,8)$ & \\
\hline Alta & 1 & $(7,7)$ & 4 & $(30,8)$ & 5 & $(19,2)$ & \\
\hline
\end{tabular}

La escala siguiente de Desviación psicopática, sigue mostrando un número bajo de individuos (dos hombres y cuatro mujeres) con conductas antisociales y ausencia de consideración hacia la mayoría de las normas sociales y morales de conducta. En cuanto a la escala Paranoia cuyo contenido refleja tanto una marcada sensibilidad interpersonal como una tendencia a mal interpretar los motivos o intenciones de los demás, los resultados muestran una baja tasa de personas transexuales con puntuaciones clínicamente significativas (tres hombres y dos mujeres).

La escala Psicastenia que refleja preocupaciones obsesivas, rituales compulsivos, miedos, ansiedad, rigidez, perfeccionismo, sentimientos de culpa e indecisión, refleja en la misma línea a lo expresado por las escalas anteriores, que hay un pequeño número de sujetos que presenta puntuaciones clínicamente significativas (tres hombres y tres mujeres).

La escala Esquizofrenia donde el contenido de los ítems cubre un amplio rango de pensamientos extraños, experiencias inusuales y características especiales, como es el conflicto de identidad sexual con sus sentimientos de inadecuación a su rol y el deseo de adquirir las características primarias y secundarias del sexo deseado, refleja artificiosamente que algunos individuos alcancen puntuaciones clínicamente significativas pero que, en realidad no cumplen criterios de tras- torno psicótico. Aun así solamente tres hombres y tres mujeres muestran perfiles clínicamente significativos.

La novena escala corresponde a la medición de Hipomanía, cuyos ítems cubren algunas de las características conductuales del trastorno bipolar tipo maníaco y que por los resultados que refleja el análisis de los datos, vemos que tal y como ocurre con las escalas precedentes hay solamente un pequeño número de sujetos que alcanzan puntuaciones clínicamente significativas (dos hombres y tres mujeres).

Finalmente, la escala Introversión social cuyas puntuaciones elevadas reflejan niveles altos de reserva social, preferencia por actividades solitarias y ausencia de asertividad, muestra resultados en consonancia con la variable Extroversión medida mediante el Inventario de Personalidad EPI de Eysenck (1987), cuyos resultados se han presentando en la tabla precedente . Concretamente el $30,8 \%$ de la muestra total alcanza niveles clínicamente significativos, con igual distribución entre sexos.

\section{DISCUSIÓN}

La exclusión de la atención sanitaria del transexualismo de la cartera de servicios del sistema sanitario público español, excepción de la 
Tabla 6. Factores de personalidad (II) según sexo biológico (MMPI-2)

\begin{tabular}{|c|c|c|c|c|c|c|c|}
\hline \multirow[t]{2}{*}{ Variables } & \multicolumn{2}{|c|}{ Hombres } & \multicolumn{2}{|c|}{ Mujeres } & \multicolumn{2}{|c|}{ Total } & \multirow[b]{2}{*}{$\chi^{2}$} \\
\hline & $N$ & $(\%)$ & $N$ & $(\%)$ & $N$ & $(\%)$ & \\
\hline Hipocondría & & & & & & & 0,41 \\
\hline Normal & 12 & $(92,3)$ & 11 & $(84,6)$ & 23 & $(88,5)$ & \\
\hline Clínicamente significativa & 1 & $(7,7)$ & 2 & $(15,4)$ & 3 & $(11,5)$ & \\
\hline Depresión & & & & & & & 0,69 \\
\hline Normal & 12 & $(92,3)$ & 11 & $(84,6)$ & 23 & $(88,5)$ & \\
\hline Clínicamente significativa & 1 & $(7,7)$ & 2 & $(15,4)$ & 3 & $(11,5)$ & \\
\hline Histeria & & & & & & & 0,21 \\
\hline Normal & 11 & $(84,6)$ & 11 & $(84,6)$ & 22 & $(84,6)$ & \\
\hline Clínicamente significativa & 2 & $(15,4)$ & 2 & $(15,4)$ & 4 & $(15,4)$ & \\
\hline Desviación psicopática & & & & & & & 0,93 \\
\hline Normal & 11 & $(84,6)$ & 9 & $(69,2)$ & 20 & $(76,9)$ & \\
\hline Clínicamente significativa & 2 & $(15,4)$ & 4 & $(30,8)$ & 6 & $(23,1)$ & \\
\hline Masculinidad-feminidad & & & & & & & 3,88 \\
\hline Normal & 2 & $(15,4)$ & 6 & $(46,2)$ & 8 & $(30,8)$ & \\
\hline Clínicamente significativa & 11 & $(84,6)$ & 7 & $(53,8)$ & 18 & $(69,2)$ & \\
\hline Paranoia & & & & & & & 0,62 \\
\hline Normal & 10 & $(76,9)$ & 11 & $(84,6)$ & 21 & $(80,7)$ & \\
\hline Clínicamente significativa & 3 & $(23,1)$ & 2 & $(15,4)$ & 5 & $(19,2)$ & \\
\hline Psicastenia & & & & & & & 3,3 \\
\hline Normal & 10 & $(76,9)$ & 10 & $(76,9)$ & 20 & $(77,0)$ & \\
\hline Clínicamente significativa & 3 & $(23,1)$ & 3 & $(23,1)$ & 6 & $(23,1)$ & \\
\hline Esquizofrenia & & & & & & & 1,81 \\
\hline Normal & 10 & $(76,9)$ & 10 & $(76,9)$ & 20 & $(77,0)$ & \\
\hline Clínicamente significativa & 3 & $(23,1)$ & 3 & $(23,1)$ & 6 & $(23,1)$ & \\
\hline Hipomanía & & & & & & & 0,29 \\
\hline Normal & 11 & $(84,6)$ & 10 & $(76,9)$ & 21 & $(80,7)$ & \\
\hline Clínicamente significativa & 2 & $(15,4)$ & 3 & $(23,1)$ & 5 & $(19,2)$ & \\
\hline Introversión social & & & & & & & 0,68 \\
\hline Normal & 9 & $(69,2)$ & 9 & $(69,2)$ & 18 & $(69,2)$ & \\
\hline Clínicamente significativa & 4 & $(30,8)$ & 4 & $(30,8)$ & 8 & $(30,8)$ & \\
\hline
\end{tabular}

Comunidad Autonómica de Andalucía que aprobó dicha prestación sanitaria en 1999, creando una unidad multidisciplinar específica en el hospital Carlos Haya de Málaga (Soriguer, 2001), está condicionando una ausencia de datos epidemiológicos, escasez de publicaciones, pocos estudios sobre comorbilidad mental asociada, sobre evolución e identificación de factores de pronóstico y sobre nivel de calidad de vida. Además y muy importante, la recomendación, expresada desde los países que llevan más tiempo trabajando este problema, de que el tratamiento se realice mediante equipos multidisciplinares, no siempre se lleva a cabo dada la inexistencia de tales equipos, excepción ya comentada, del ejemplo andaluz. 


\section{Variables biográficas}

La prevalencia del transexualismo en España, a excepción de Andalucía, es desconocida, debido a la exclusión de la atención sanitaria pública ya mencionada. Los datos de los que disponemos proceden de publicaciones procedentes de países occidentales como Alemania, Suecia, Holanda, Inglaterra, Estados Unidos y Australia, las tasas son muy variables yendo desde $1 / 2.900$ a $1 / 100.000$ para los transexuales hombre a mujer y desde $1 / 8.300$ a $1 / 400.000$ para los transexuales mujer a hombre. Llama la atención la discrepancia de casos que hay entre sexos, con mayor incidencia en hombres biológicos pero también, marcadas diferencias entre países. La razón hombre/mujer oscila entre 2/1 en Alemania y 6.1/1 en Australia, siendo la proporción media de tres hombres por cada mujer (Landén, Walinder y Lunströn, 1996; Weitze y Osburg, 1996; Garrels et al, 2000).

Los estudios de incidencias muestran que no han sido estables a lo largo del tiempo y, que hay diferencias marcadas entre muestras publicadas por distintos países, mientras que en Suecia, en una revisión de tres décadas (19722002), la proporción entre sexos cambió de $1 / 1$ a principio de los años 70 hasta casi $2 / 1$ a favor de los transexuales hombre a mujer en los años 90 (Olsson y Moller, 2003). En cambio, en Alemania la proporción entre sexos permaneció constante de $2 / 1$ a favor de los transexuales hombre a mujer desde 1970 a 1994 , pero a partir de esa fecha hasta el 2000 la proporción entre sexos tendió a igualarse, siendo de 1.2/1 todavía a favor de los transexuales hombre a mujer (Garrels et al, 2000).

La tendencia a la igualación del número de casos entre sexos ha sido total en nuestra muestra de estudio, puesto que el número de transexuales hombre a mujer que solicitó tratamiento para el cambio de sexo ha sido igual al número de transexuales mujer a hombre, siendo, por consiguiente, la razón entre sexos 1/1.

En los últimos años el número de casos de transexualismo parece incrementarse. Este aumento de prevalencia parece deberse a una mejora en los tratamientos disponibles y a la mayor aceptación sociocultural del trastorno, lo que conlleva a que el número de personas que solicitan terapia sea mayor (Gómez et al, 2003).

La edad media de solicitud de reasignación sexual es de 20-25 años para el grupo mujer a hombre y de 25-30 años para el grupo hombremujer (Van Kesteren, Gooren y Megens, 1996). Datos similares aportan Olsson y Moller (2003) en la muestra Sueca de estudio, donde encuentran que actualmente la diferencia de edad ente sexos es de 6 años mayores de edad, como promedio, los transexuales hombre a mujer que a la inversa.

Nuestros datos apuntan en la misma dirección que los resultados precedentes, al situarse la edad media para la muestra de transexuales de ambos sexos en los mismos rangos de edad (25 años para las mujeres biológicas y 28,92 años para los hombres biológicos). Por otra parte, se mantiene la desigualdad de edades entre sexos a la hora de demandar la terapia de reasignación sexual, ya que los transexuales hombre a mujer tienen una edad media superior en tres años a los transexuales mujer a hombre.

\section{Consecuencias psicosociales, conductas de riesgo y agresiones sociales}

Existe un enraizado patrón de discriminación y prejuicio contra las personas transexuales en nuestra sociedad. Debido a ello suelen experimentar discriminación económica al tener dificultades para conseguir un trabajo, tanto por su baja cualificación laboral derivada de una interrupción temprana de la escolarización como por el rechazo que sufren, sobre todo los transexuales hombre a mujer (Blanchard, 1985; Bodlund y Kullgren, 1996), por parte de ciertos empleadores con actitud misógina y con desconfianza, que podría ser el resultado de la existencia de un clima social extenso que sanciona severamente a las personas que no se adaptan a las normas sociales referentes al género.

Una de las dificultades más importantes que tienen que afrontar es el aislamiento social que en muchos caso padecen, por disponer de una red de apoyo social muy precaria derivada de la estigmatización que sufren, al tener un autocon- 
cepto poco reforzado socialmente (Cohen-Kettenis y Van Goozen, 1997) y que contribuye a crear dificultades de adaptación social, escolar y laboral, conduciendo en muchos casos, sobre todo de transexuales hombre a mujer, a la realización de trabajos marginales, ilegales o peligrosos como la prostitución y la realización de conductas de riesgo (sexo sin preservativo, coito anal, uso de drogas por vía endovenosa), que condicionan seriamente su salud física y mental, con alta prevalencia de VIH/SIDA y hepatitis B y C (Belza et al, 2000; Clements-Nolle, Marx, Guzman y Katz, 2001; Harcourt, van Beek, Heslop, McMahon y Donovan, 2001; Spizzichino et al, 2001; Vester, Davoli, Camposeragna, Valeri y Perucci, 2001; Kenagy, 2002).

Se ha constatado en diversos estudios (Haraldsen y Dahl, 2000; Cano y Bergero, 2003), a que las personas transexuales que tienen la posibilidad de realizar los tratamientos de reasignación sexual y que son apoyados emocionalmente por la familia, especialmente por la madre, muestran un nivel bajo de conductas de riesgo y de síntomas psicopatológicos, tanto antes como después del tratamiento. En cambio, los padres que reaccionan de forma culpabilizadora, aumentan la vulnerabilidad, que ya de por sí padecen debido a su condición, aumentando la posibilidad de que aparezcan episodios depresivos e intentos de suicidio (Aguado, Ruíz y Mas, 2001).

Aparte de la mencionada discriminación económica, padecen de forma frecuente experiencias de violencia. Lombardi, Wilchins, Prising y Malouf (2001) sobre una muestra de 402 casos, encontró que la mitad de las personas transexuales había experimentado algún tipo de violencia o acoso a lo largo de su vida y una cuarta parte había experimentado algún incidente violento. Datos que se confirman en nuestra muestra, puesto que la mitad de la misma manifestó haber sufrido agresiones verbales en el entorno social y laboral, casi la mitad $(46,2 \%)$ de los transexuales hombre a mujer había sufrido agresiones físicas y el 25,4\% agresiones sexuales en su mayoría realizadas por extraños, frente al 7,7\% de los transexuales mujer a hombre que había sufrido agresiones físicas y ninguno agresiones sexuales. Por consiguiente, la discriminación y prejuicios que se mantienen contra la población transexual se manifiesta con mayor intensidad hacia los transexuales femeninas (hombre a mujer).

\section{Salud mental}

Las dificultades que encuentran para ser tratados por el sistema sanitario público y el sufrimiento que les produce el sentimiento de disforia de género, les lleva, en determinados casos, a padecer problemas psicológicos diversos. En cambio, las personas transexuales que tienen la posibilidad de realizar el cambio de sexo muestran un nivel relativamente bajo de síntomas o de trastornos psicopatológicos tanto antes como después de los tratamientos irreversibles (Haraldsen y Dahl, 2000). Datos que no apoyan las conclusiones aportada por algunos autores sobre que el transexualismo por si mismo está asociado con severos trastornos de la personalidad (Miach, Berach, Butcher y Rouse, 2000).

Actualmente hay un cuestionamiento acerca de la consideración del transexualismo como un trastorno mental severo y, asimismo, aparecen en la literatura controversias sobre la presencia de una elevada frecuencia de patología mental grave asociada (Hales, Yudofsky y Talbott, 1996; Cole, O`boyle y Emory, 1977; Roberts y Gotlib, 1997; Becerra, de Luis y Piedrola, 1999; Haraldsen y Dahl, 2000; Miach et al., 2000; Kersting et al, 2003).

En los estudios que sustentan que padecen comorbilidad mental añadida al transexualismo, los trastornos mentales asociados que con mayor frecuencia padecen son: trastornos de personalidad (narcisista, antisocial y límite), trastornos depresivos, trastornos de ansiedad, trastornos psicosomáticos, esquizofrenia, conductas autodestructivas y abuso de sustancias psicoactivas (Hales et al., 1996; Roberts y Gotlib, 1997; Becerra et al., 1999).

Ahora bien, la comorbilidad se muestra diferente según el sexo biológico, siendo tanto más frecuente en hombres biológicos (transexuales hombre a mujer) como también expresando diferencias, a favor de los transexuales mujer a hombre, en cuanto a la evolución y al pronóstico (Hepp, Klaghofer, Burkhard-Kubler y Buddeberg, 2002). Son los trastornos de la perso- 
nalidad y la esquizofrenia los que están asociados a una peor evolución del trastorno de identidad de género (Caspari, Sttinger y Lang, 1999).

En nuestra muestra de estudio los resultados sobre la salud mental reflejan una baja frecuencia de patología mental asociada. Solamente hay siete $(26,9 \%)$ transexuales de la muestra con niveles moderados de depresión y diez $(38,5 \%)$ con niveles altos de ansiedad, cuyos niveles podrían deberse a la experimentación de estrés alto en los últimos doce meses, como consecuencia del proceso de decisión de iniciar el tratamiento de cambio de sexo, el tiempo de espera para poder ser atendidos, la búsqueda de apoyo y de recursos económicos para hacer frente a los tratamientos hormonales y quirúrgicos.

Se sabe que el contar con apoyo social es un buen predictor para afrontar problemas en general, pero particularmente el proceso de cambio de sexo por las connotaciones sociales negativas que tiene en nuestra sociedad, comprobamos que la mayoría de nuestra muestra $(69,2 \%)$ cuenta con buenos niveles de apoyo, hecho que se hace evidente en sus altos niveles de autoestima (61,5\% de la muestra total).

En cuanto a los datos sobre los factores de personalidad, la mitad de la muestra global tiene nivel alto de ansiedad rasgo y tendencia a la inestabilidad emocional, lo que predispone a padecer trastornos psicológicos ante situaciones estresantes y que como ha sido descrito con anterioridad, queda reflejado en los niveles de ansiedad y de depresión mostrados.

Trastornos graves de la personalidad o de la salud mental no hemos encontrado, puesto que el porcentaje de transexuales (entre un $11,5 \%$ y $23,1 \%$ sin distinción de sexo) que alcanzó puntuaciones clínicamente significativas en algunas de las variables, eran debidas a un efecto artificial producido por la propia prueba de personalidad, al detectar como patológicos pensamientos típicos de los conflictos de identidad de género, por las dificultades para acceder a tratamiento ya mencionadas y por las consecuencias psicosociales añadidas y suficientemente explicadas.

No hemos encontrados apenas diferencias entre sexos biológicos en la expresión de los trastornos asociados, salvo en Neuroticismo donde los hombres biológicos muestran en un $69,2 \%$ altos niveles frente al $23,1 \%$ de las mujeres biológicas. Lo contrario ocurre en el miedo ante las situaciones sociales donde se pudiera descubrir el sexo biológico, ya que aquí mientras todas las mujeres biológicas (transexuales masculinos) tienen ese miedo, para el grupo de transexuales femeninos (hombre a mujer) alcanza al 46,1\%.

\section{Posibles limitaciones del estudio, indicaciones $y$ directrices para futuras investigaciones}

El tamaño de la muestra no es muy numeroso, pero como ha sido reflejado en los datos de prevalencia publicados, la frecuencia del trastorno es baja y desconocida en nuestro país, por haber estado excluida su atención de la cartera de servicios del sistema nacional de salud. Los primeros resultados obtenidos han revelado una baja frecuencia de patología mental asociada y perfiles normales de personalidad, pero con niveles altos de estrés debido posiblemente tanto al propio proceso de decisión de iniciar el tratamiento de cambio de sexo, al tiempo de espera para poder iniciarlo y a la búsqueda de apoyos y recursos. Sería necesario ampliar el estudio con el fin de reunir una muestra más amplia de personas transexuales, para poder generalizar con mayor seguridad los resultados obtenidos sobre su salud mental y, también, analizar con más profundidad los factores causales o asociados a los problemas de salud mental que manifiestan, con la finalidad de poder intervenir de una forma más eficaz.

\section{REFERENCIAS}

Aguado, J., Ruíz, M.A., y Mas, B. (2001). Abusos sexuales a menores. Variables mediadoras y psicopatología asociada. Psicologemas, 15, 5-42.

American Psychiatric Association (2002). Manual diagnóstico y estadístico de los trastornos mentales DSMIV-TR. Barcelona: Masson.

Becerra, A., de Luis, A., y Piedrola, G. (1999). Morbilidad en pacientes transexuales con auto-tratamiento 
hormonal para cambio de sexo. Medicina Clínica, 113, 484-487.

Beck, A.T., Rush, A.J., Shaw, B.F., y Emery, G. (1979). Cognitive therapy of depression. Nueva York: Guilford Press.

Belza, M.J., Llacer, A., Mora, R., de la Fuente, L., Castilla, J., Noguer, J., y Canellas, S. (2000). Social characteristics and risk behaviors for HIV in a group of transvestites and male transsexuals engaging in street prostitution. Gaceta Sanitaria, 14, 330-337.

Blanchard, R. (1985). Typology of male-to-female transsexualism. Archives of Sexual Behavior, 14, 247-261.

Bodlund, O., y Kullgren, G. (1996). Transsexualism- general outcome and prognostic factors: A five-year followup study of nineteen transsexuals in the process of changing sex. Archives of Sexual Behavior, 25, 303-316.

Bower, H. (2001). The gender identity disorder in the DSM-IV classification: a critical evaluation. Australian and New Zealand Journal of Psychiatry, 35, 1-8.

Butler, G., Cullington, A., Munby, M., Amies, P.L., y Gelder, M.G. (1984). Exposure and anxiety management in the treatment of social phobia. Journal of Consulting and Clinical Psychology, 52, 642-650.

Calanca, A. (1991). Le transsexual après le changement. Evolution et pronostic. Helvetica Chirurgica Acta, 58, 257-260.

Cano, C., y Bergero, T. (2003). Intervención psicológica en una unidad de trastornos de la identidad de género: un desafío sanitario. En E. Remor, P. Arranz y S. U1l (Eds.), El psicólogo en el ámbito hospitalario (pp. 729745). Bilbao: Desclée De Brouwer.

Caspari, D., Sittinger, H., y Lang, B. (1999). Transsexualism and schizophrenic psychosis. Problems preparing expert opinion on transsexualism. Psychiatry Prax, 26, 89-92.

Clements-Nolle, K., Marx, R., Guzman, R., y Katz, M. (2001). HIV prevalence, risk behaviors, health care use, and mental health status of transgender persons: implications for public health intervention. American Journal of Public Health, 91, 915-921.

Cohen-Kettenis, P.T., y van Goozen, S. (1997). Sex reassignment of adolescents transseuxals: a follow-up study. Journal of American Academy and Child Adolescents Psychiatry, 36, 263-271.

Cole, C.M., O’boyle, M., y Emory, L.E. (1997). Comorbidity of gender dysphoria and other major psychiatric diagnoses. Archives of Sexual Behavior, 26, 13-26.

Conde, V., y Franch, J.I. (1984). Escalas de evaluación comportamental para la cuantificación de la sintomatología psicopatológica en los trastornos angustiosos y depresivos. Madrid: Laboratorios Upjohn.

Echeburúa, E., y Corral, P. (1998). Manual de violencia familiar. Madrid: Siglo XXI.

Eysenck, H.J., y Eysenck, S.B.G. (1964). Eysenck Personality Inventory. Londres: Hodder and Stoughton, Educational. (Versión española, TEA ediciones, 1987).
Garrels, L., Kockott, G., Michael, N., Preuss, W., Renter, K., Schmidt, G., Sigusch, V., y Windgassen, K. (2000). Sex ratio of transsexuals in Germany: the development over three decades. Acta Psychiatrica Scandinavica, 102, 445-448.

Gómez, M., Solá, E., Garzón, S., García, S., Cubells, P., y Hernández, A. (2003). Aproximación epidemiológica a los trastornos de identidad de género. En A. BecerraFernández. Transexualidad. La búsqueda de una identidad (pp. 59-63). Madrid: Ediciones Díaz de Santos.

Hales, R.E., Yudofsky, S.C., y Talbott, L.E. (1996). The American Psychiatric Press. Tratado de Psiquiatría. Barcelona: Ancora.

Haraldsen, I.R., y Dahl, A.A. (2000). Symptom profiles of gender dysphoric patients of transsexual type compared to patients with personality disorders and healthy adults. Acta Psychiatrica Scandinavica, 102, 276-281.

Harcourt, C., van Beek, I., Heslop, J., McMahon, M., y Donovan, B. (2001). The health and welfare needs of female and transgender street workers in New South Wales. Australian and New Zealand Journal of Public Health, 25, 84-89.

Harry Benjamin International Gender Dysphoria Association (HBIGDA) (1998). The Standars of care for Gender Identity Disorders ( $5^{a}$ version). Mineapolis: HBIGDA.

Harry Benjamin International Gender Dysphoria Association (HBIGDA) (2001). The Standars of care for Gender Identity Disorders ( $6^{a}$ version). Mineapolis: HBIGDA.

Hathaway, S.R., y McKinley, J.C. (Adaptación española: Ávila-Espada, A y Jiménez-Gómez F). (2000). Inventario Multifásico de Personalidad de Minnesota - 2 . Madrid: TEA ediciones.

Heimberg, R.G., Dodge, C.S., Hope, D.A., Kennedy, C.R., Zollo, L.J., y Becker, R.E. (1990). Cognitive-behavioral group treatment for social phobia: Comparison to a credible placebo control. Cognitive Therapy and Research, 14, 1-23.

Hengstschläger, M., van Trotsenburg, M., Repa, C., Marton, E.; Huber, J.C., y Bernaschek, G. (2003). Sex chromosome aberrations and transsexualism. Fertility and Sterility, 79, 639-640.

Hepp, U., Klaghofer, R., Burkhard-Kubler, R., y Buddeberg, C. (2002). Treatment follow-up of transsexual patients. A catamnestic study. Nervenarzt, 73, 283-288.

Kenagy, G.P. (2002). HIV among transgendered people. AIDS Care, 14, 127-134.

Kersting, A., Reutemann, M., Gast, U., Ohrmann, P., Suslow, T., Michael, N., y Arolt, V. (2003). Journal Nervous and Mental Disease, 191, 182-189.

Landén, M., Walinder, J., y Lunströn, B. (1996). Prevalence, incidence and sex ratio of transsexualism. Acta Psychiatrica Scandinavica, 93, 221-223. 
Lombardi, E.L., Wilchins, R.A., Priesing, D., y Malouf, D. (2001). Gender violence: transgender experiences with violence and discrimination. Journal of Homosexuality, 42, 89-101.

Mattick, R.P., Peters, L., y Clarke, J. (1989). Exposure and cognitive restructuring for social phobia: A controlled study. Behavior Therapy, 20, 3-23.

Miach, P.P., Berach, E.F., Butcher, J.N., y Rouse, S. (2000). Utility of the MMPI-2 in assessing gender dysphoric patients. Journal of Personality Assesment, 75, 268-279.

Michel, A., Mormont, C., y Legros, J.J. (2001). A psychoendocrinological overview of transsexualism. European Journal of Endocrinology, 145, 365-376.

Miguel, J.J, y Cano, A.R. (1986). Inventario de Situaciones y Respuestas de Ansiedad. Madrid: TEA ediciones.

Monstrey, S., Hoebeke, P., Dhont, M., De Cuypere, G., Rubens, R., Moerman, M., Hamdi, M., Van Landuyt, K., y Blondeel, P. (2001). Surgical therapy in transsexual patients: a multi-disciplinary approach. Acta Chrirurgica Belgique, 101, 200-209.

Olsson, S.E., y Moller, A.R. (2003). On the incidence and sex ratio of transsexualism in Sweden, 1972-2002. Archives of Sexual Behavior, 32, 381-386.

Oganización Mundial de la Salud (OMS) (1992). Trastornos mentales y del comportamiento. Descripciones clinicas y pautas para el diagnóstico. Madrid: Meditor.

Rakic, Z., Starcevic, V., Maric, J., y Kelin, K. (1996). The outcome of sex reassignment surgry in Belgrade: 32 patients of both sexes. Archives of Sexual Behavior, 25, 515-525.

Rehman, J., Lazer, S., Benet, A., Schaefer, L., y Melman, A. (1999). The reported sex and surgery satisfactions of 28 postoperative male-to-female transsexual patients. Archives of Sexual Behavior, 28, 71-89.

Roberts, J.E., y Gotlib, I.H. (1997). Lifetime episodes of dysphoria: gender, early childhood loss and personality. British Journal Clinical Psychology, 36, 195-208.

Rosenberg, M. (1965). Society and the adolescent self image. Princeton: Princeton University Press.

Snaith, P., Tarsh, M.J., y Reid, R. (1993). Sex reassignment surgery. A study of 141 Dutch transsexuals. British Journal of Psychiatry, 162, 681-685.
Soriguer, F.J.C. (2001). La atención a los transexuales en España. A la búsqueda del tiempo perdido. Cirugía Plástica Ibero-Latinoamericana, 27, 259-262.

Spielberger, C.D., Gorsuch, R.L. y Lushene, R.E. (1970). Manual for the State/Trait Anxiety Inventory. Palo Alto, CA: Consulting Psychologist Press (Versión española, TEA, 1982).

Spizzichino, L., Zaccarelli, M., Rezza, G., Ippolito, G., Antinori, A., y Gattari, P. (2001). HIV infection among foreign transsexual sex workers in Rome: prevalence, behavior patterns, and seroconversion rates. Sexual Transmission of Disease, 28, 405-411.

Tangpricha, V., Ducharme, S.H., Barber, T.W., y Chipkin, S.R. (2003). Endocrinologic treatment of gender idnetity disorders. Endocrinology Practice, 9, 12-21.

Van Kesteren, T.J., Gooren, L.J., y Megens, J.A. (1996). An epidemiological and demographic study of transsexuals in The Netherlands. Archives of Sexual Behavior, 25, 589-600.

Vester, A., Davoli, M., Camposeragna, A., Valeri, C., y Perucci, C.A. (2001). Prevalence of HIV infection and risk behaviour among street prostitutes in Rome, 19971998. AIDS Care, 13, 367-372.

Vogt, J.H. (1968). Five cases of transsexualism in females. Acta Psychiatrica Scandinavica, 44, 62-88.

Walinder, J., Lundström, B., y Thuwe, I. (1978). Prognostic factors in the assessment of male transsexuals for sex reasignment. British Journal of Psychiatry, 132, 16-20.

Walinder, J., y Thuwe. I. (1975). A social-psychiatric follow-up study of 24 sex-reassigned transsexuals. Reports from the Psychiatric Center, St Jorgen's Hospital, University of Göteborg, Göteborg.

Ward, R.A. (1977). Aging group consciousness. Implications in a older sample. Sociology and Social Research, 61, 496-519.

Watson, D., y Friend, R. (1969). Measurement of SocialEvaluative Anxiety. Journal of Consulting and Clinical Psychology, 33, 448-457.

Weitze, C., y Osburg, S. (1996). Transsexualism in Germany: empirical data on epidemiology and application of the Germans transsexuals'act during its first years. Archives of Sexual Behavior, 25, 409-425. 